\title{
Optical SAR Images Fusion: Comparative Analysis of Resulting Images Data
}

\author{
Yuhendra*, Minarni \\ Institut Teknologi Padang, Informatics Engineering Department, Padang, Indonesia,
}

\begin{abstract}
Image fusion is a useful tool for integrating low spatial resolution multispectral (MS) images with a high spatial resolution panchromatic (PAN) image, thus producing a high resolution multispectral image for better understanding of the observed earth surface. A main proposed the research were the effectiveness of different image fusion methods while filtering methods added to speckle suppression in synthetic aperture radar (SAR) images. The quality assessment of the filtering fused image implemented by statistical parameter namely mean, standard deviation, bias, universal index quality image (UIQI) and root mean squared error (RMSE). In order to test the robustness of the image quality, either speckle noise (Gamma map filter) is intentionally added to the fused image. When comparing and testing result, Gram Scmidth (GS) methods have shown better results for good colour reproduction, as compared with high pass filtering (HPF). And the other hands, GS, and wavelet intensity hue saturation (W-IHS) have shown the preserving good colour with original image for Landsat TM data.
\end{abstract}

\section{Introduction}

Currently, following with increasing the rapid advancements of new and greatly improved various space born imaging sensor systems in Remote Sensing (RS), it is can be done by both active (SAR, LiDAR) and passive (optical and thermal range, multispectral and hyper-spectral) devices and applied in many area studying Earth observational applications [1]. A lower spatial resolution multispectral (MS) image and high spatial resolution panchromatic (PAN) imaging sensors are the systems usually used for earth change observation phenomena, each one having its own specific advantage. Almost all of the operating earth observation very high resolution (VHR) imagery (Spot, Landsat, Ikonos, WorldView, QuicBird, GeoEye, and Orbview, etc), it was very important issues in various RS problems application in image sharpening, land classification, change detection, and object identification, visualization purposes etc. [2]. Otherwise, PAN imaging sensors provides an image in the visible band, which is characterized by high spatial information content well suited for intermediate scale mapping applications and urban analysis [2]. Considering image fusion observed scene analysis and RS application, the observed scene information fusion can be requirement in the following cases [3]; data recorded by different sensors (multi-sensor image fusion), data recorded by the same sensor scanning the same scene at different dates (multi-temporal image fusion), data recorded by the same sensor operating in different spectral bands (multi-frequency image fusion), data recorded by the same sensor at different polarizations (multi-polarization image fusion), and data recorded by the same sensor located on platforms flying at different heights (multi-resolution image fusion). A Multi-sensor, multi-temporal, multi-resolution and multi-parameter image data from operational Earth observation satellites are available and therefore possibly give a more complete view of observed objects [4]. Increasing the interest of area, some researcher has addressed multitemporal data fusion problem. Some publication interest on the multi-sensor image fusion and application has reported [3-9]. The development of the multi-sensor information fusion is fundamental for change detection purpose but can be also valuable in image classification to improve the classification accuracy. The Principal study of fusing multiple images provided by heterogeneous image sensor has been addressed in many literatures with different methods, context and application [10].

In the synthetic aperture radar (SAR) images, speckle is coherent interference of waves scattered from terrain elements observed in each resolution cell with spatial resolution greater than the wavelength. It's can be reduced by incoherent processes. Due to the roughness of the imaged surface, each resolution cell will contain several scatterers, and the resulting image will have a granular appearance due to constructive and destructive interference. Speckle appears as spatially correlated, multiplicative noise that is statistically independent of the image intensity, although it is a radiometric feature of the imaged object.

Concerning used numerous methods and application for image fusion and filter fused in remotes sensing, it is

\footnotetext{
* Corresponding author: yuhendra $@$ ieee.com
} 
desirable to give a general assessment of the quality of the fused images filtering from the viewpoint of practical use. In this research, we use merging images fusion methods to assess the capability of fusion multi sensor using Wavelet-IHS (intensity-hue-saturation), HPF, and Grand Schmidt while image added to speckle noised, eg. Gamma Map filters (Figure 1) to observe of fused image for better spectral quality in object identification. Another commons investigation, we apply the following statistical parameter approaches for standardizing and automating the evaluation process of the fused images. First, we have examine the qualitative assessment is made with conventional visual inspection.

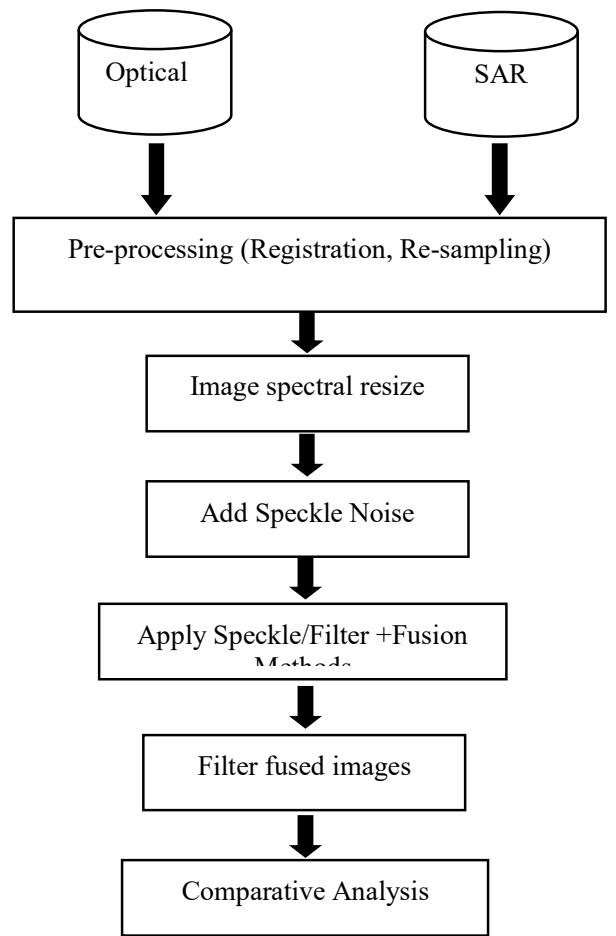

Fig. 1. Flowchart of filtering fused images

Table 1. Characteristics of Optical SAR Sensors

\begin{tabular}{|c|c|c|c|}
\hline Sensor & Band Name & $\begin{array}{l}\text { Wavelength } \\
(\mu \mathrm{m})\end{array}$ & $\begin{array}{c}\text { Resolution } \\
\text { (m) }\end{array}$ \\
\hline \multirow{6}{*}{ LANDSAT } & B1 & $0.45-0.52$ & \multirow{6}{*}{$30 \mathrm{~m}$} \\
\hline & $\mathrm{B} 2$ & $0.52-0.60$ & \\
\hline & B3 & 0.63-0.69 & \\
\hline & B4 & $0.76-0.90$ & \\
\hline & B5 & $1.55-1.75$ & \\
\hline & B7 & $2.08-2.35$ & \\
\hline SAR & $\mathrm{L}$ & $\begin{array}{c}\mathrm{HH} \\
\text { Polarization }\end{array}$ & $12,5 \mathrm{~m}$ \\
\hline
\end{tabular}

\section{Study Area and Satellite Imagey}

\subsection{Study Area}

The study site for this work is located in the Sultan Hasanuddin International Airport in Makassar, South Sulawesi, Indonesia, see in Fig.2.

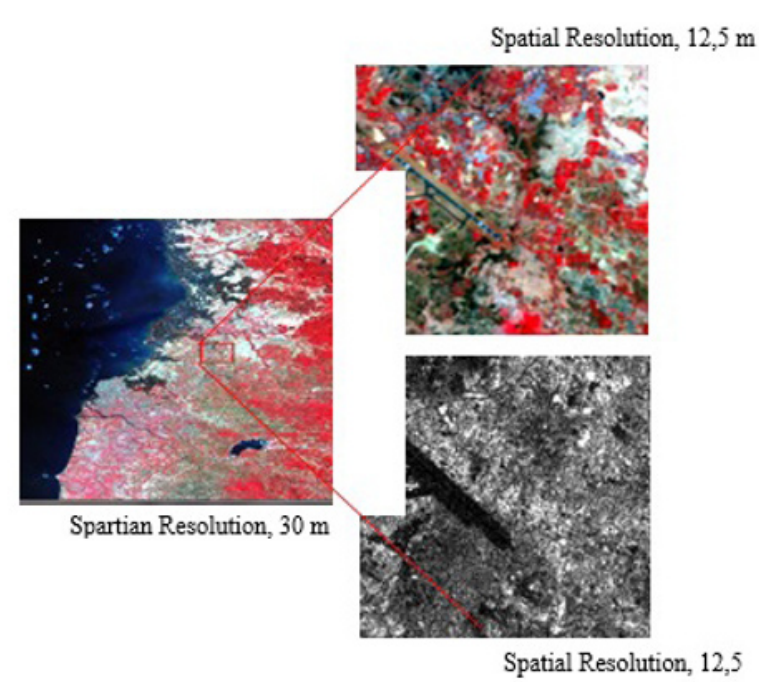

Fig. 2. Subset study area in South Sulawesi, Makasar

\subsection{Satellite Imagery}

For this work, two optical SAR images acquired by Lansat-5 TM and JERS-1 acquired on September 28, 2002 and August 18, 1998 with path row 116/64, 85/309 respectively, were used for investigating the performance of optical SAR image fusion analysis. A Lansat-5 images consists of one PAN and five MS with a spatial resolution of $30 \mathrm{~m}$ and SAR image has 2,5 spatial resolution with $\mathrm{L}$ Band, $\mathrm{HH}$ Polarization. The characteristics of both images are summarized in Table 1.

\section{Methods}

\subsection{Pre-processing}

\subsubsection{Image correction and registration}

The most important prerequisite for accurate data fusion is precise geometric correction. In image fusion needs commons control point on both the input images since different images of the same area used together. The common geometric correction is image to image registration. Registration can be done in various methods. A one of methods is image to image registration. An image to image registration is translation and rotation alignment process by which two images of like geometry on the same geographic area [12]. In registration processing, the most accurate way is to register the input images separately by establishing geometric relationship between the image and the ground using rigorous photogrammetric methods [13].

\subsubsection{Re-sampling:}

Next, we apply re-sampling, in which each data point (pixel) in the high-resolution base map is assigned with a value based on the MS image pixels. In order to achieve a good fusion result, low spatial spectral images should be re-sampled. At present, nearest neighbour resampling is often adopted which has some effects on the precision of new image. In this paper, an image fusion method is proposed with Cubic Convolution technique. In this way, Optical-SAR images with $30 \mathrm{~m}$ and 12,5 
spatial resolutions are produced from original LandsatTM and JERS-1 images, respectively (Figure 2).

\subsubsection{Spatial resolution ratio:}

The spatial resolution ratio improvement can make a sharpen image while preserving spectral information contained in the fused images. Since PAN images usually have a better spatial resolution than the multispectral images of the same sensor, while the MS images provide spectral properties of the objects. In image fusion, spatial resolution ratio plays an important role in image fusion.

\subsubsection{Speckle (Image filtering):}

Speckle is a scattering phenomenon and not a noise. The discrimination of different natural media by comparing intensity to a fixed threshold leads, in general to numerous errors due to the high variability of SAR speckled response. There are various speckle reduction filters available in image processing to process SAR images. Some give better visual interpretations while others have good noise reduction or smoothing capabilities. The use of each filter depends on the specification for a particular application. In image processing, the standard speckle filters like Median, Mean, Statistical Lee, Kuan, Frost, enhanced Frost and Gamma filters are generally used. Each of these speckle filters performs the filtering based on either local statistical data given in the filter window to determine the noise variance within the filter window. The analysis of image we used Gamma MAP filter. It was based on the assumption that the (un-speckled) intensity of the underlying scene is gamma distributed. The filter minimizes the loss of texture information better than the Frost and Lee filters within gamma distributed scenes. It is suitable for a wide range of gamma distributed scenes, such as forested areas, agriculture areas, and oceans.

\subsection{Image Processing Using Fusion Algorithm}

\subsubsection{Gram-Schmidt (GS)}

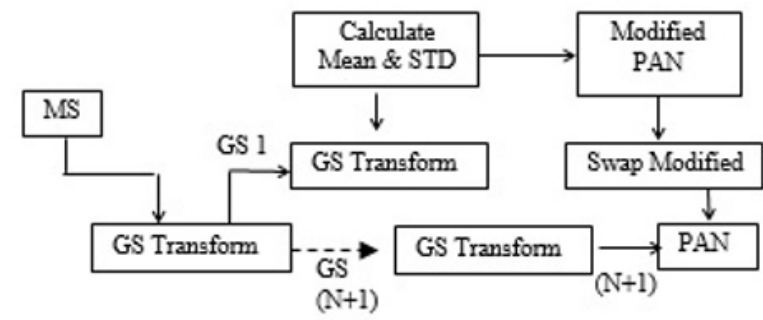

Fig. 3. GS Fusion Technique

\subsubsection{High Pass Filter}

The HPF technique is based on injecting spatial details taken from the PAN image onto the re-sampled MS images (Schowengerdt, 1998). The mathematical model can be given as

$$
F_{i, j}=M S_{i, j}+\left[P A N_{i, j}-\overline{P A N}_{i, j(w, h)}\right]
$$

where $F_{i, j}$ is the pixel of the fused image at coordinate $(i$, $j), M S_{i, j}$ and $P A N_{i, j}$ are corresponding pixel values in MS and PAN image, respectively; and $\overline{P A N}_{i, j}(w, h)$ stands for the local mean of high-resolution channel inside the window of $w$ (width) $\times h$ (height) pixels centered at $(i, j)$.

\subsubsection{Wavelet-HIS}

The Wavelet transform is a mathematical tool that is extensively used in image analysis and image fusion. The basic idea of image fusion, based on two-band wavelet transformation, is that a low-resolution image is replaced by the low frequency portion of the image in a two-band wavelet transformation. In W-PCA, the wavelet is transformed into principal components, and the first principal component is fused with the PI. Then, inverse wavelet transform is applied to the fused principal component and remaining principal components to obtain the fused image.

\subsubsection{Statistical Parameter}

Commonly used the statistical parameter, quality of fused image was assessed by means of some quality measures that can predict perceived image quality automatically. In this work, two types of quality assessment approaches are taken, namely qualitative (visual analysis and quantitative methods. The visual analysis is based on the visual comparison of the color between the original MIs and the fused image and that of the spatial details between the original PI and the fused image. These methods inevitably involve subjective factors and personal preference that can influence the result of evaluation. While visual inspection has limitation due to human judgment, quantitative approach based on the evaluation of "distortion" that is brought about by the noise added to the fused image is more desirable for mathematical modeling [18]. In this work, a validation method is proposed based on a quality criterion, namely, Mean, Standard Deviation, Bias, UIQI , Root mean squared error (RMSE) [6]] to estimation and chosen as a robustness criterion. This statistical parameter is often used for evaluation of fusion techniques. These parameters are defined as follows:

- Mean value refers to the gray level of pixels in an image. If the image size is $m \times n$, the mean value is defined as

$$
\text { Mean }=\frac{1}{m n} \sum_{i=j}^{m} \sum_{j=1}^{n} x_{i j} \text {, }
$$

- Standard deviation (STD) reflects the deviation degree of values relative to the mean of the image. Normally, the STD is defined as

$$
S T D=\left[\frac{1}{n-1} \sum_{i=1}\left(M S_{i, j}-M S_{\text {mean }}\right)^{2}\right]^{1 / 2}
$$


Bias is the difference between the mean of the original image and that of the fused image. The ideal value is zero:

$$
\text { Bias }=\frac{M S_{\text {mean }}-\text { fused }_{\text {mean }}}{M S_{\text {mean }}}=1-\frac{\text { fused }_{\text {mean }}}{M S_{\text {mean }}}
$$

- $R M S E$ is the root mean square error computed as

$$
\operatorname{RMSE}\left(B_{i}\right)=\operatorname{Bias}^{2}\left(B_{i}\right)+\operatorname{STD}^{2}\left(B_{i}\right)
$$

- Universal image quality index (UIQI) is used to measure the similarity between two images.

$$
U I Q I=\frac{\sigma_{A B}}{\sigma_{A} \sigma_{B}} \cdot \frac{2 \mu_{A} \mu_{B}}{\mu_{A}^{2}+\mu_{B}^{2}} \cdot \frac{2 \sigma_{A} \sigma_{B}}{\sigma_{A}^{2}+\sigma_{B}^{2}}
$$

\section{Results and Discussion}

Figure 4a-b show the original Landsat-TM PAN and JERS-1 image with Gamma MP filtering added. Both of images has a same spatial resolution $12,5 \mathrm{~m}$ has after resampling that can be employed into fused image filter, see Figure 5.
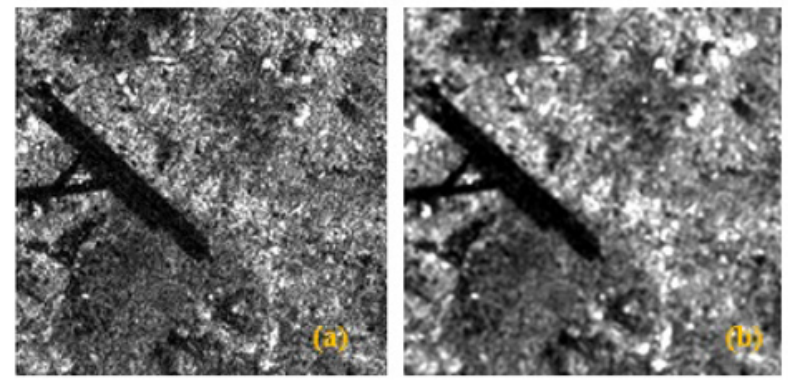

Fig. 4. Image filter before fused, (a) Original image, (b) Gamma MP
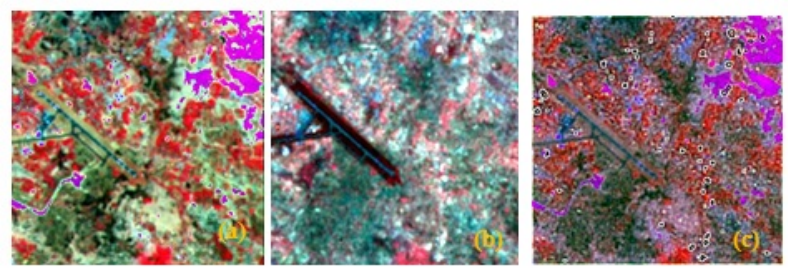

Fig. 5. Fused image filter, (a) Gamma MP-Wavelet IHS, (b) Gamma MP-Gram Schmidt, (c) Gamma MP-HPF

A thorough examination of spectral quality is made for filtering fused images using the following seven statistical parameters: mean, bias, STD, RMSE, Cov, and UIQI. The fused image that best preserves the original spectral information, and hence exhibits the highest spectral quality, can be characterized with the following conditions: (i) the mean and SDT values are closer to those of the original MS images, (ii) the smallest values for the error parameters (RMSE, Bias, UIQI). Tables III summarize the results of the present analysis. The highest value has been obtained in the GS-fused image since closer with original image and smaller bias. Thus, the GS method produces the result that has the least color distortion as a whole and the best preservation of spectral characteristics. In the universal index quality image
(UIQI) is evaluated to study the similarity between the originally fused image and each of the filtering-added images. A UIQI index is considers the similarity of two images on the basis of three different factors, namely, loss of correlation, radiometric distortion, and contrast distortion. A Wavelet-HIS has a higher value.

Table 2. Inter Band Correlation of Filter Fused Images

\begin{tabular}{|l|c|c|c|}
\hline \multicolumn{1}{|c|}{ Methods } & Band 1 \&2 & Band 1 \& 3 & Band 2 \& 3 \\
\hline Original MS & 0.925 & 0.866 & 0.966 \\
Wavelet-IHS & -0.079 & 0.529 & -0.158 \\
Gram Schmidt & 0.920 & 0.858 & 0.965 \\
HPF & 0.352 & 0.743 & 0.373 \\
\hline
\end{tabular}

As another test, we can examine the inter-band correlation that should be preserved in image fusion. Table 2 shows the comparison of the inter-band correlations (B1/B2, B1/B3, and B2/B3), among the original optical image and between the original and fused SAR images. The highest correlation around 0.920 and 0.965 were seen for the combination of Band $1 / 2$ and Band $2 / 3$, irrespective of the fusion method employed. The results in Table 3 show that both GS give values reasonably in agreement with those in the original MS images.

\section{Conclusions}

We have compared to combine Speckle Filter with Fusion methods to Integrating Optical \& SAR image. Based on spectral quality assessment (Mean, STD \& Cov) GS followed by PCA are for best, closet to original optical image value. The spectral analyses (Bias, DIV, RMSE) of the filter fused images indicate that the results generated with GS, Wavelet HIS, and HPF methods out performance preserve spectral and spatial information.

\section{Acknowledgment}

We would gratefully acknowledge like to thanks the anonymous reviewers for valuable comments and suggestions

\section{References}

1. Yuhendra, Joshapat T.S., Assessment of multi temporal image fusion for remote sensing application, International Journal on advance science engineering information technology, 7(3), 778-784, 2017

2. Zeng, Y., Zhang, J., Van Genderen, J.L., Zhang, Y., Image fusion for land cover change detection, Int. J. Image and Data Fusion, 1(2), 193-215. 2010

3. Chibani, Y., 2007, Integration of panchromatic and SAR features into multispectral SPOT images using the 'a trous' wavelet decomposition. Int. J. of Remote Sensing, 28, 2295-2307.

4. Zhu, L., Tateishi, R., 2006. Fusion of multi-sensor multi temporal satellite data for land cover mapping, Int. J. Remote Sensing, 27(5), 903-918. 
5. Cao, G., Hou, P., Ya-Qiu, J., Xianqiang, M., 2009. Fusion of features in multi-temporal SAR imagery to detect changes I urban areas, Int. J. Remote Sensing, 30(20), 5989-6001

6. Ehler M, Klonus S, Astrand P.J, Rosso P. Multisensor for pansharpening in remote sensing, International Journal Image and Data Fusion.2010;1,1, 25-45

7. Molch, K., 2010. Multi-sensor and multi-temporal fusion of VHR satellite imagery based on KIM, IEEE Geoscience and Remote Sensing Letter, 7(1).

8. Kandrika, S., Ravinsankar, T., 2011. Multi-temporal satellite imagery and data fusion for improved land cover information ectraction, Int. J. Image and Data Fusion, 2, 1, 61-73.

9. Wurm, M., Taubenbock, H., Schardt, M., 2011. Object-based image information fusion using multi sensor earth observation data over urban areas, Int. J. Image and Data Fusion, 2,2, 121-147.

10. Yuhendra, Alimuddin, I., Josaphat Tetuko, S.S., Kuze, H., 2012. Assessment of pan-sharpening methods applied to image fusion of remotely sensed multi-band data, Int. J. of Applied Earth Observation \& Geoinformation, 18,165-175.

11. Otazu X, González M, Fors O, Núñez J. Introduction of Sensor Spectral Response Into Image Fusion Methods. Application to Wavelet-Based Methods, IEEE Transactions on Geoscience \&Remote Sensing.2005;43(10), 2376-2385.

12. Chen, L., Lee, L., 1992. Progressive generation of control framework for image registration, Photogrammetric Engineering \& Remote Sensing, 58(9):1321-1328.

13. Lee C, Bethel J, 2001. Georegistration of Airborne Hyperspectral Image Data. IEEE Trans. on Geoscience and Remote Sensing. 39(7): 1347-1351.
14. Choi M. A new intensity-hue-saturation fusion approach to image fusion with a tradeoff parameter, IEEE Trans. Geosciences Remote Sensing.2006;2 44 1672-1682.

15. Karathanassi V, Kolokousi P, Ionnidou S. A comparison study on fusion methods using evaluation indicators, Int. J. Remote Sensing.2007; 28(10), 2309-2341.

16. Ling Y, Ehler M, Usery E.L, Madden M. FFTenhanced IHS transform method for fusing highresolution satellite images, ISPRS Journal of Photogrammetry \& Remote Sensing. 2007; 61, 381392

17. Kumar U, Mukhopadhyay C, Ramachandra T.V. Pixel based fusion using IKONOS imagery, Int. J. Recent Trend in Engineering. 2009; 1(1),173-175.

18. Bovolo F, Bruzzone L, Capobianco L, Garzelli A, Marchesi S. Analysis of effect of pan-sharpening in change detection, IEEE Geoscine. Remote Sensing Letter. 2010; 7(1)

19. Shah V. P, Younan N. H, and King R. L. An efficient pansharpening method via a combined adaptive PCA approach and contourlets. IEEE Trans. Geosciences Remote Sensing.2008; 4.

20. Kumar U, Mukhopadhyay C, Ramachandra T.V. Pixel based fusion using IKONOS imagery, International Journal Recent Trend in Engineering.2009;1(1),173-175.

21. Wang L, Cao X, Chen J. ISVR: an improve synthetic variable ratio method for image fusion, Geocarto International.2008; 23(2), 155-165.

22. Rahman M.M, Sri Sumantyo J.T, Sadek M.F. Microwave and optical image fusion for surface and sub-surface feature mapping in Eastern Sahara, International Journal Remote Sensing. 2010; 31(20), $5465-5480$

Table 3. Quality Index Fused images with Gamma map filtering

\begin{tabular}{|c|c|c|c|c|c|c|c|c|}
\hline \multirow{2}{*}{ Methods } & \multicolumn{8}{|c|}{ Index } \\
\hline & Band & Mean & STD & Cov & Bias & DIV & RMS & UIQI \\
\hline \multirow{3}{*}{ Original Image } & $\mathrm{R}$ & 68 & 9 & 14 & - & - & - & - \\
\hline & $\mathrm{G}$ & 96 & 6 & 79 & - & - & - & - \\
\hline & $\mathrm{B}$ & 83 & 8 & 46 & - & - & - & - \\
\hline \multirow{3}{*}{ Wavelet-IHS } & $\mathrm{R}$ & 68 & 10 & 53 & 0 & -2.785 & 1 & 0.87 \\
\hline & $\mathrm{G}$ & 89 & 24 & -18 & 0.073 & 1.227 & 18.0 & 0.93 \\
\hline & $\mathrm{B}$ & 82 & 9 & 91 & 0.012 & -0.978 & 1 & 0.80 \\
\hline \multirow{3}{*}{ Gram Schmidt } & $\mathrm{R}$ & 68 & 11 & 27 & 0 & -0.928 & 11 & 0.49 \\
\hline & $\mathrm{G}$ & 96 & 16 & 78 & 0 & 0.012 & 16 & 0.44 \\
\hline & $\mathrm{B}$ & 83 & 9 & 47 & 0 & -0.021 & 9 & 0.46 \\
\hline \multirow{3}{*}{ HFP } & $\mathrm{R}$ & 68 & 9 & 61 & 0 & -3.35 & 0 & 0.86 \\
\hline & $\mathrm{G}$ & 86 & 33 & 101 & 0.104 & -0.27 & 27 & 0.80 \\
\hline & $\mathrm{B}$ & 83 & 8 & 75 & 0.180 & -0.63 & 0.18 & 0.65 \\
\hline
\end{tabular}

